\title{
An Approach to Face Detection and Feature Extraction using Canny Method
}

\author{
Ranjana Sikarwar \\ Department of CSE, \\ SAIT, Jabalpur
}

\author{
Pradeep Yadav \\ Department of CSE, \\ ITM GOI, Gwalior
}

\begin{abstract}
This paper presents a hybrid approach to face detection and feature extraction. The remarkable advancement in technology has enhanced the use of more accurate and precise methods to detect faces. This paper presents a combination of three well known algorithms Viola- Jones face detection framework, Neural Networks and Canny edge detection method to detect face in static images. The proposed work emphasizes on the face detection and identification using Viola-Jones algorithm which is a real time face detection system. Neural Networks will be used as a classifier between faces and non-faces. Canny edge detection method is an efficient method for detecting boundaries on a face in this proposed work. The Canny edge detector is primarily useful to locate sharp intensity changes and to find object boundaries in an image.
\end{abstract}

\section{Keywords}

\section{MLFFN, FAR, FFR, HIT RATE, CANNY}

\section{INTRODUCTION}

Face detection plays an important role in the field of image processing and biometric systems which are being used most widely nowadays in a number of real-world applications. A great variety of work has been carried out by making use of artificial neural networks (ANN) in combination with one or more the face detection algorithms in the field of image processing and pattern recognition. Face detection means to determine if there are actually any human faces present in an image. Exact localization of faces and facial features within grey scale images is a challenging task due to the high inconsistency, in both shape and texture, of the look of the human face. The process of face detection in images is complex because of variations present in and around the facial images such as: pose; look; position and orientation; different skin tones; presence of bifocals or scars or marks on the faces; differences in camera gain; lighting conditions; and image resolution. The core idea behind the study of face detection and recognition came from the need of security in real-world and in biometrics. Another need may arise for the study or analysis of facial expressions by the psychologists. Face detection and recognition is used in a number of applications such as surveillance systems, passport verification, PC cameras etc. Face detection needs to be done prior to the face recognition. This is performed to filter out the useful information for face and to analyze the facial expressions. Thus relevant techniques are requisites for the representation of faces and for the extraction of facial features. A face can be represented as an array of pixels intensity values while features of the face such as eyes, nose, mouth etc can be extracted by using some geometrical-based approaches.

\section{LITERATURE REVIEW}

In 1998[3] Henry A. Rowley, Shumeet Baluja, and Takeo
Kanade, proposed a neural network-based approach for the detection of upright frontal views of the faces. Neural networks are used to invigilate small apertures of an image and decide whether each aperture contains a face. It has been observed from the experimental results that the system can detect $90.5 \%$ of the faces using a test set of 130 complex images, with an acceptable number of false positives. The system in operation works in two stages. The first stage applies some filters to an image and the second stage combines the outputs using an arbitrator. The filters continuously search for face at each area of image at different scales and then the outputs of filters are cascaded by the arbitrator also excluding overlaying detections. A fast version of the system can process a 320x240 pixel image in 2 to 4 seconds on a $200 \mathrm{MHz}$ R4400 SGI Indigo 2.

Rowley et al. [4] prepared a neural system specifically on the image pixels, to segment the picture sub windows into face/non-confront areas. This system models the face as a $20 * 20$ pixel format. Again each one extricated sub window is preprocessed utilizing lighting adjustment and histogram equalization. A Multi-Layer Perceptron (MLP) is then connected to the standardized sub window to order the locale as a face or non-confront.

In 1994 [5] Yang and Huang, presented an knowledge based method. It detects faces in three stages or levels. At first stage high level rules are applied. In second stage the histogram and equalization techniques are utilized for edge detection lastly eyes and mouth features are found. Learning based strategy presented by Kotropoulos and Pitas.

In 1995 [6] Leung, proposed face detection by matching labeled graph. Gaussian filters and distribution distance are utilized. The beginning step is to confine the facial features areas. Five facial features like two eyes, two nostrils and nose lip intersection are thought to be important to form a face.

In 1997 [7] Sung and Poggio, proposed distinguishment of face and non face classes based upon distribution distance. Positive and negative illustrations of face and non-faces examples are trained. The techniques can be isolated into two sections. Firstly the distribution-based model is proposed for separation of face and none face designs and secondly a multilayer perceptron classifier.

Schneidermann and Kanade [8] model the face patch utilizing a methodology taking into account the naive Bayes classifier. Numerous classifiers (thought to be free) are joined. Every classifier gauges the joint likelihood of neighborhood appearance and position of a facial subregion.

The system is slow, as all classifiers need to be applied to each subwindow, yet gives great results. On the CMU information set the detection rate is $94.4 \%$ with 65 false positives [37]. 
Rowley [9] [10] proposed strategy based upon multilayer neural system. Face and non-face classes are learn by the system.

\section{PROPOSED METHODOLOGY}

The approach used to detect the faces in the image and extraction of the facial gimmicks as demonstrated in fig 1.are portrayed in the accompanying sections. The first step towards proposed technique is the creation of database for images. The training for the database is done through neural network. Neural Network has a high-quality performance in face detection but takes a long time to process. Here after training it is decided whether the trained image is face or either non face. If the trained image is face then take an input image from test image for face detection. Faces are detected using viola-Jones detector which creates bounding boxes on the face region. Through edge detection method facial features are sharply extracted. After completing all these processes the canny edge detector method is applied for face feature extraction. At last after going through all these procedures the performance of facial feature extraction is improved by noise removal.

\subsection{Create database for images}

Image database created for face detection consist of 500 images for faces and 10 for non faces for our training process in different scale, pose, illumination and camera variation. Some of the images were taken from web as shown below and some were taken from web cam. The test images in a dataset are shown in figure below.
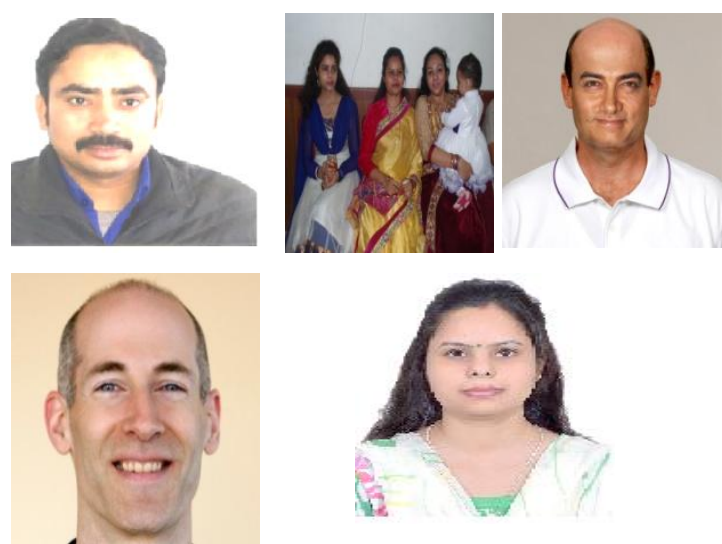

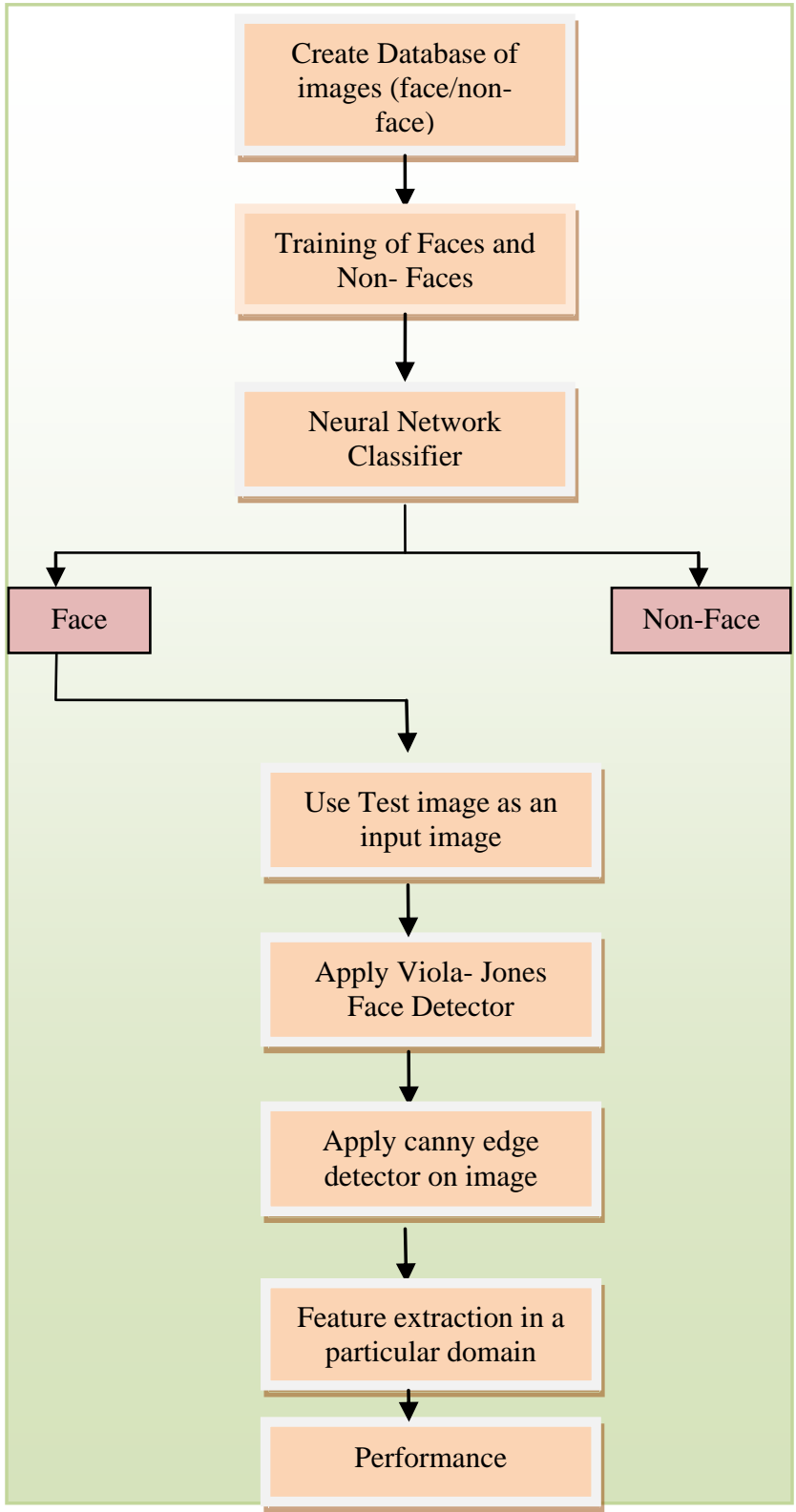

Fig.1: Block Diagram of Face Detection Approach

\subsection{Neural Network as a classifier}

The proposed methodology makes use of the customized neural network model in the MATLAB tool which is called Multi-Layer Feed- Forward which distinguishes between faces and non- faces as shown in fig. 3. The measurement of the window size is $20 \times 20$ pixels represent human appearances and non-faces, the input vector is constituted by 2160 neurons, and the concealed layer has 100 neurons.

The structural planning of neural system utilized is indicated as a part of fig 2 and 3 .

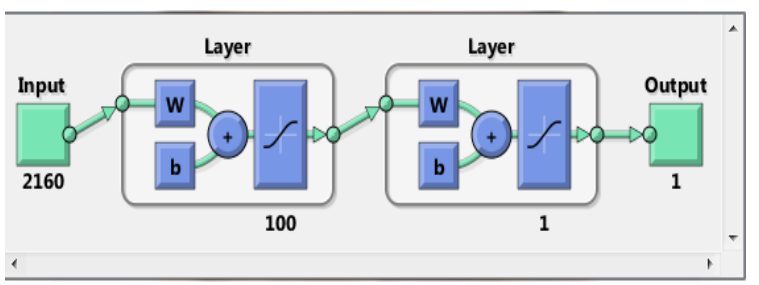

Fig.2 Neural Network Architecture 


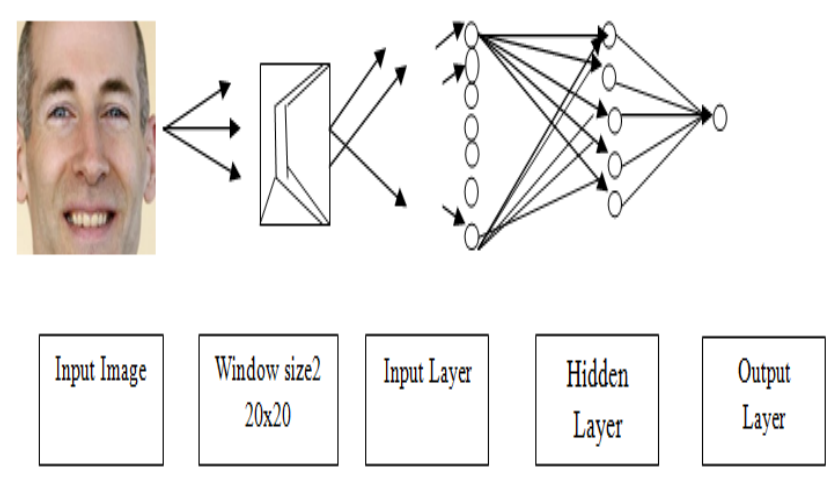

Fig.3 Structure of proposed system

\subsection{Neural Network training algorithm}

Scale Gradient Conjugate (SCG) a type of training algorithm which has shown a good level of performance in face classification. As seen from the experimental results SCG converges and reduces the error with minimum iterations as compared to other training algorithms.

The output of this classifier is then used as an input to violaJones algorithm for face detection and feature extraction.

\subsection{Apply Viola- Jones for Face detection}

The Viola- Jones face detector creates bounding boxes on face and then facial features. The cascade object detector uses the Viola - Jones algorithm to identify human faces, noses, eyes or mouth as shown in figure 4.
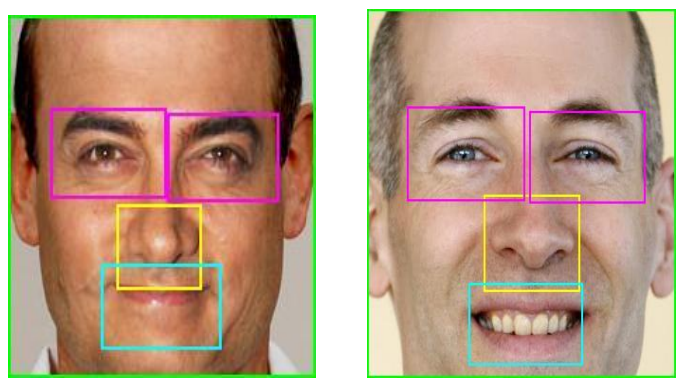

Fig. 4 Viola- Jones Face detector

\subsection{Edge detection using canny method}

After the localization of the face and facial parts of the input image, the result from the past step is consolidated with the edge of the input image. Here canny algorithm is used for edge detection. Canny method detects edges of the face more sharply and it's a promising approach as compared to Sobel edge, Robert's cross, Prewitt edge and the Laplacian of Gaussian edge. Figure 5 demonstrates the use of edge detection by canny method.

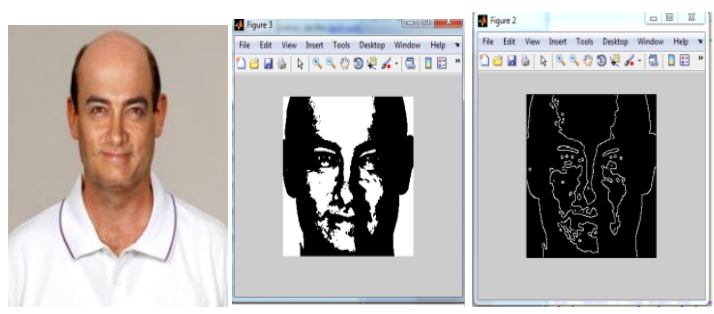

Fig 5. Edge detection: (a) Input image (b) binary (c) Canny edge image

\subsection{Proposed Algorithm}

The proposed algorithm used in this paper is used to detect upright frontal views of human faces in images accompanied by the extraction of facial features using canny method. Multilayer feed forward neural network is trained to distinguish faces and non- faces. Viola-Jones method detects faces using Haar-like features.

1. Initialize the network for training the face and nonface database. If it finds face then signifies ' 1 ' value otherwise signifies ' 0 ' for non-face.

2. Consider the test image to be resized to $\mathrm{N} \times \mathrm{N}$; (Original image I having $\mathrm{N}$ value of pixel)

3. Image is to be changed into gray and double for 2-D image.

4. The proposed algorithm detects face parts using Viola and Jones algorithm which works on Harr feature for faces.

5. The image is represented as $I € \mathrm{R}^{\wedge} \mathrm{W} \times \mathrm{H}$, where $\mathrm{W}$ and $\mathrm{H}$ represent image width and height the dimension of image space $\mathrm{W} \times \mathrm{H}$.

6. The input image set is represented as $\mathbf{I n}=\{\mathrm{I} 1, \mathrm{I} 2, \mathrm{I} 3$, I4....In\}.

7. The selected features for the image $I$ are $F n=\{F 1$, $F 2, F 3, F 4 \ldots F N)$.

8. The attribute set is defined as $A \mathrm{i}=\{\mathrm{A} 1, \mathrm{~A} 2$, $\mathrm{A} 3 \ldots A \mathrm{n}\}$.where $\mathrm{A} 1, \mathrm{~A} 2 \ldots$. An represent attributes of the image.

9. Create bounding box for particular object using cascade object detection function.

10. A bbox is the bounding box which contains a four element vector, [x y height width] that specifies in pixels.

11. Calculate image region on the basis of standard size. Take size of the image in terms of bbox.

12. Sorts the matrix based on the rows specified in the vector row. Flip the matrix up to down.

13. Find ratio of an image based on the above calculated region.

14. The Shape Inserter object draws rectangles, lines, polygons, or circles on images.

15. Find edges of an image using canny method for all face parts.

\section{RESULT ANALYSIS}

MATLAB R2012A tool is used to carry out the experimental results. We have used face images from web as shown in fig. 1 and database for the experiment. Graph1 shows the training of the multilayer feed forward network. Graph 2 shows the overall result of the proposed system for face detection and feature extraction. The system shows accurate results for the upright frontal faces with good illumination condition. Figure1 in Graph1 shows the work of the viola- Jones face detector creating bounding boxes around the face while figure 2 shows the result of the canny edge detector which sharply draws the edge boundaries on the facial region.

Table1 shows the data of 5 images including Num of Faces, Num of Hits, Num of missed faces, Run time and accuracy in 
given image. Accuracy is calculated in terms of false positive rate and false negative rate. The False positive rate and false negative rate which depends upon the number of correct faces missed and false faces detected correctly and number of incorrectly detected face respectively.

The accuracy is calculated by the equation below [18].

\section{Accuracy $($ in $\%)=100$-(false positive rate + false negative rate)}

Table 1. Computation of Face Detection Performance

\begin{tabular}{|c|c|c|c|c|c|}
\hline Images & $\begin{array}{l}\text { Nu } \\
\mathrm{m} \text { of } \\
\text { face } \\
\mathrm{S}\end{array}$ & $\begin{array}{l}\text { Hit } \\
\text { Rate }\end{array}$ & $\begin{array}{l}\text { Miss } \\
\text { Rate }\end{array}$ & $\begin{array}{l}\text { Run } \\
\text { time(sec) }\end{array}$ & $\begin{array}{l}\text { Accurac } \\
\mathbf{y}\end{array}$ \\
\hline$\underset{\mathrm{g}}{\text { Image1.jp }}$ & 1 & $\begin{array}{l}3.499 \\
7\end{array}$ & $\begin{array}{l}1.883 \\
6\end{array}$ & $\begin{array}{l}14.34358 \\
7\end{array}$ & 94.6167 \\
\hline $\begin{array}{c}\text { Image2.jp } \\
\mathrm{g}\end{array}$ & 1 & $\begin{array}{l}3.358 \\
3\end{array}$ & $\begin{array}{l}1.707 \\
7\end{array}$ & $\begin{array}{l}16.26546 \\
2\end{array}$ & 94.9340 \\
\hline $\begin{array}{l}\text { Image3.jp } \\
\mathrm{g}\end{array}$ & 1 & $\begin{array}{l}3.358 \\
3\end{array}$ & $\begin{array}{l}1.707 \\
7\end{array}$ & $\begin{array}{l}28.33650 \\
7\end{array}$ & 94.9340 \\
\hline $\begin{array}{c}\text { Image4.jp } \\
\mathrm{g}\end{array}$ & 1 & $\begin{array}{l}3.358 \\
3\end{array}$ & $\begin{array}{l}1.707 \\
7\end{array}$ & 15.52813 & 94.9340 \\
\hline $\begin{array}{c}\text { Image5.jp } \\
\text { g }\end{array}$ & 1 & $\begin{array}{l}3.358 \\
3\end{array}$ & $\begin{array}{l}1.707 \\
7\end{array}$ & $\begin{array}{l}20.05230 \\
1\end{array}$ & 94.9340 \\
\hline
\end{tabular}

Table 2 below compares the result of the proposed system using viola-Jones and canny edge detector with the one using Adaboost and neural network [1].

As shown in the Table2 accuracy of proposed system is $94 \%$ while that of Base system (Neural Network+ Adaboost) is of $93.4 \%$. Since the base system is trained and implemented on grayscale images, thus the processing is faster with grayscale images. While in our proposed system we have used color images still we can see that accuracy is slightly better than the base system because of not using the Adaboost algorithm as it produces more high false positive rate [1]

Table 2. Comparison of Face Detection Performance

\begin{tabular}{|c|c|c|}
\hline & Detection rate & $\begin{array}{c}\text { False } \\
\text { positive rate }\end{array}$ \\
\hline $\begin{array}{c}\text { Proposed } \\
\text { System }\end{array}$ & $94 \%$ & $3-4 \%$ \\
\hline $\begin{array}{c}\text { Neural Networkwith } \\
\text { Adaboost }\end{array}$ & $93.34 \%$ & $0.34 \%$ \\
\hline
\end{tabular}

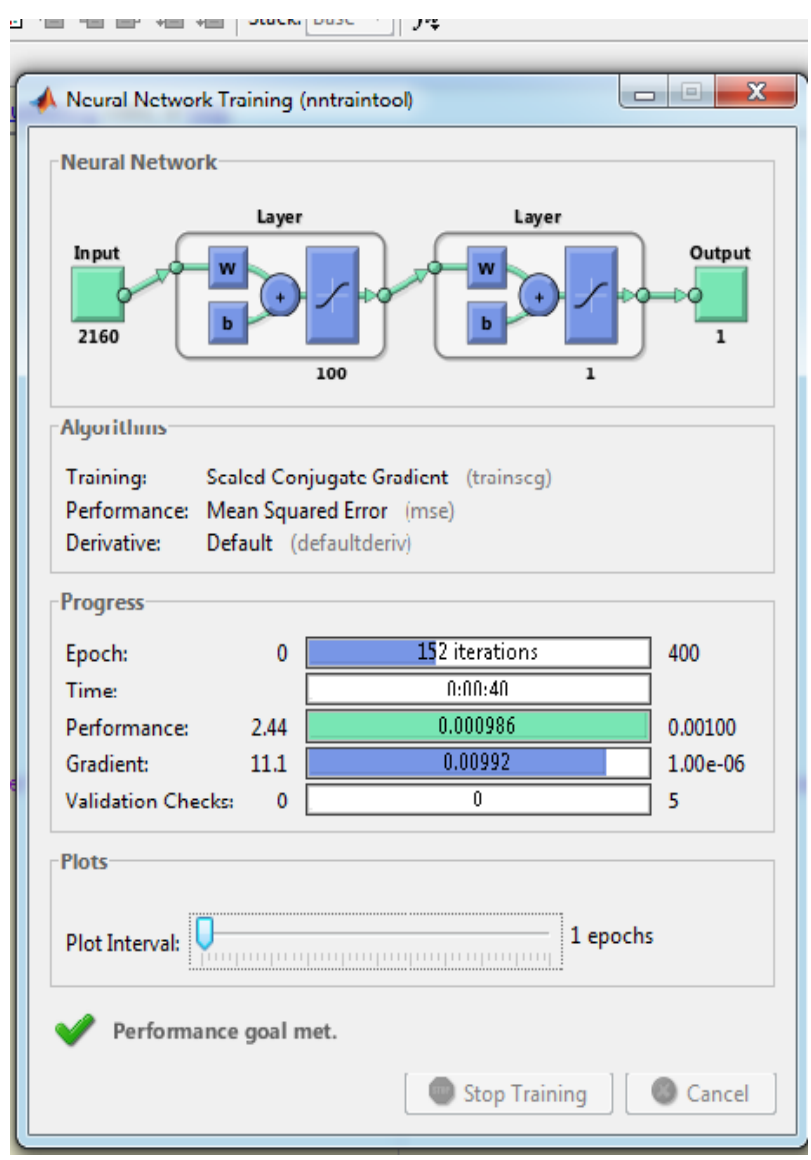

Graph1. Neural Network training tool

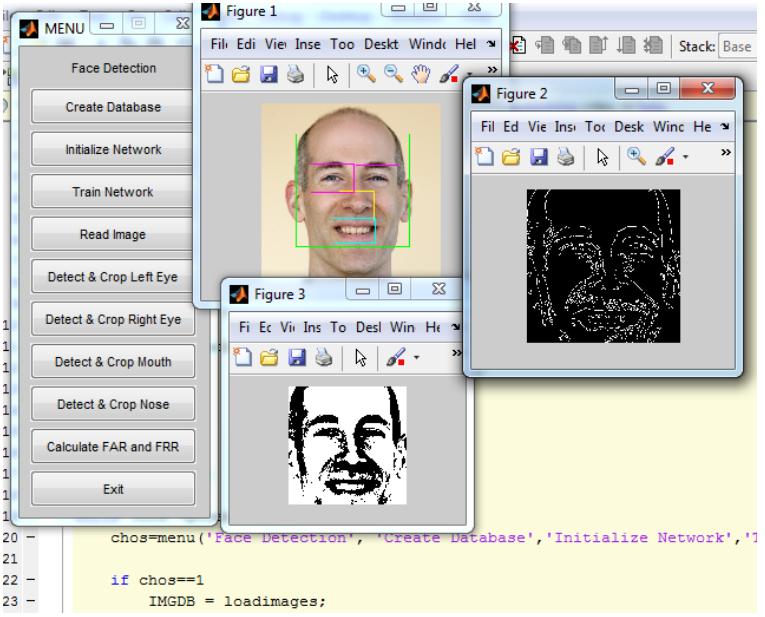

Graph2. Performance graph of proposed system

\section{CONCLUSION}

This paper presented an approach which is a combination of three well known algorithms for face detection in human images. Initially the experiments were carried out only on a few numbers of upright frontal views of human faces in order to check the feasibility of the procedure and to decrease the training time of neural networks. In the later phases of the experiments a larger number of test images were used compromising with the execution time but producing accurate results than the one used with Adaboost algorithm for face detection. Also using Adaboost produces high false positive rates. Moreover if the histogram equalization is used to improve the image quality before testing, it is expected to produce much better results. In future this algorithm can be 
applied to detect human faces at multi-view points as well; currently it is restricted to upright frontal views of the face only. Instead of taking only $2 \mathrm{D}$ images we will implement the later work using 3D images.

\section{REFERENCES}

[1] Zulhadi Zakaria"Face Detection Using Combination of Neural Network and Adaboost" Intelligent Biometric Group School of Electrical and Electronics Engineering Universiti Sains Malaysia.

[2] S.P.Khandait, Dr. R.C.Thool, "Hybrid Skin Detection Algorithm for Face localization in facial Expression Recognition", Proceedings of international conference on Advance computing conference-09 (IACC- 09), Patiala, Punjab, India, 6-7 March'09.

[3] Henry A. Rowley, Shumeet Baluja, and Takeo Kanade. Neural network based face detection. IEEE Trans. Pattern Anal. Mach. Intell., 20(1):23-38, 1998.

[4] H. A. Rowley, S. Baluja, and T. Kanade. Human face detection in visual scenes. In D. S. Touretzky, M. C. Mozer, and M. E. Hasselmo, editors, Advances in Neural Information Processing Systems, volume 8, pages 875881. The MIT Press, 1996.

[5] Kotropoulos, C. Pitas, I, "Rule-based face detection in frontal views" Acoustics, Speech, and Signal Processing, 1997. ICASSP-97., 1997 IEEE International Conference on Volume 4, 21-24 April 1997 Page(s):2537 - 2540 vol.4.

[6] Leung, T.K.; Burl, M.C.; Perona, P., "Finding faces in cluttered scenes using random labeled graph matching",Computer Vision, 1995. Proceedings., Fifth International Conference on 20-23 June 1995 Page(s):637 - 644

[7] Sung,K.-K, Poggio T, "Example based learning for view based human face detection" Pattern Analysis and Machine Intelligence, IEEE transactions on, volume 0,Issue 1 Jan 1998, Pages( 39 - 51).

[8] H. Schneiderman and T.Kanade. Probablistic modelling of local appearance and spatial relationships for object recognition. In Computer Vision and Pattern Recognition Conference 1998, 1998.
[9] Rowley, HA:, Baluja, S:, Kanade, T:, "Neural NetworkBased face Detection"Computer Vision and Pattern Recognition, 1996 Proceedings CVPR96, 1996 IEEE Computer Society Conference on, 18-20 June 1996, pages 203-208.

[10] Rowley, HA:, Baluja, S:, Kanade, T:, "Rotation invariant Neural Network-Based face Detection"Computer Vision and Pattern Recognition, 1998. Proceedings. 1998 IEEE Computer Society Conference on 23-25 June 1998 Page(s):963 - 963.

[11]http://homepages.inf.ed.ac.uk/rbf/CVonline/low/edges/can ny.htm S Price, "Edges: The Canny Edge Detector", July 4, 1996.

[12] Cristinacce, D., Cootes, T., and Scott, I. (2004). A multistage approach to facial feature detection. In 15th British Machine Vision Conference, 231-240.

[13] X. Wang and X. Tang, "Face Photo-Sketch Synthesis and Recognition," IEEE Transactions on Pattern Analysis and Machine Intelligence (PAMI), Vol. 31, 2009.

[14]Extract of Facial Feature Point, IJCSNS International Journal of Computer Science and Network Security, VOL.9 No.1, January 2009

[15] Neural Network Based Approach for Face Detection cum Face Recognition, World Academy of Science, Engineering and Technology.

[16] Face detection, Inseong Kim, Joon Hyung Shim, and Jinkyu Yang.

[17] Robust Face Detection Method Based on Skin Color and Edges, J Inf Process Syst, Vol.9, No.1, March 2013. [18] Detecting Boundaries for Segmentation and Recognition

[18] Phil Brimblecombe (2002) "Face Detection using Neural Networks", H615 - Meng Electronic Engineering, School of Electronics and Physical Sciences, URN: 1046063.

[19] Lixin Fan kah-kay sung, "Model -based varying pose face detection and facial feature registration in color images" 2002. 